\title{
Interne und externe Faktoren der Verschuldung Mexikos ${ }^{\star}$
}

\section{Fragestellung}

Die Schuldenkrise der Dritten Welt darf, neben der globalen Ökologiefrage, als größte Herausforderung der neunziger Jahre an die Weltwirtschaft bezeichnet werden. Die Entwicklung des Nord-SüdVerhältnisses wird wesentlich von der Lösung dieser beiden Fragenkomplexe abhängen. In diesem Zusammenhang muß es von Interesse sein, sich mit den Ursachen der Schuldenkrise auseinanderzusetzen. Nur eine klare Ursachenanalyse setzt uns in die Lage, über die bloße Symptombekämpfung hinaus das Übel an der Wurzel zu packen. Außerdem ist das Verständnis der Ursachen unumgänglich, wenn wir eine Wiederholung der Schuldenkrise vermeiden wollen. Befürchtungen drängen sich vor allem angesichts der immensen Kapitalien auf, mit welchen die rasche Entwicklung Osteuropas erzwungen werden soll.

Das Fallbeispiel Mexiko ist aus zwei Gründen bedeutsam. Erstens war Mexiko 1982 der Auslöser für eine umfassende Schuldenkrise vieler - nicht aller! - Drittweltstaaten, die nun schon beinahe ein Jahrzehnt anhält. Zweitens ist Mexiko mit über 100 Mrd. \$ Auslandschulden nach wie vor hinter Brasilien das am zweithöchsten verschuldete Entwicklungsland (EL).

Mexiko ist ein von der Natur reich ausgestattetes Land. So bekannte Agrarprodukte wie Chili, Tomaten, Avocados und Mais haben ihre Heimat in Mexiko. Zudem findet man in Mexiko umfangreiche Rohstoffvorkommen. Bei Silber, Blei, Zinn, Kupfer, Mangan, Schwefel, Flußspat und Erdöl gehört Mexiko mit zu den führenden Produzentenländern. Die nachgewiesenen Erdölreserven betrugen Mitte der achtziger Jahre über $70 \mathrm{Mrd}$. Faß. Die potentiellen Reserven von $200 \mathrm{Mrd}$. Faß werden nur noch von denjenigen der Sowjetunion übertroffen $(\mathrm{NOH}-$ LEN, 1984, 398). Wie konnte es geschehen, daß Mexiko trotz dieser zahlreichen natürlichen Ressourcen in eine so mißliche wirtschaftliche Lage geriet, wie sie die Verschuldungskrise vom Herbst 1982 offenlegte? Und sind die Mexikaner am Debakel tatsächlich selber schuld? Etwas wissenschaftlicher

*Der Artikel basiert auf einem Gastvortrag des Autors an der TU Berlin am 20. November 1989. ausgedrückt: Sind die Verschuldungsprobleme Mexikos intern oder extern verursacht? In dieser Form des simplen «Entweder-oder» ist die Frage allerdings falsch gestellt und läßt sich nicht beantworten. Im folgenden geht es dem Autor darum, sowohl die internen als auch die externen Ursachen der mexikanischen Schuldenprobleme und ihr komplexes Zusammenspiel aufzuzeigen.

\section{Verschuldung und Entwicklung}

Unter Verschuldung versteht man im Zusammenhang mit der Dritten Welt üblicherweise die Außenverschuldung. Sie läßt sich als rückzahlbarer Teil des Kapitalimports definieren. Das Gegenstück zu dieser externen Verschuldung bildet die interne Verschuldung oder Binnenschuld. Bei Verschuldungszahlen ist außerdem zu beachten, ob man von der kurz- oder langfristigen Perspektive ausgeht, ob die öffentlichen oder privaten Schulden gemeint sind und ob man das Brutto- oder Nettokonzept zugrunde legt.

Die Verschuldung von Entwicklungsländern wird von manchen Kreisen generell als schädlich, d. h. entwicklungshemmend gehalten. Der Autor teilt diese Ansicht nicht. A priori sollte man Verschuldung weder als positiv noch als negativ einstufen. Neben den seit 1982 gehäuften Fällen, in welchen die Aufnahme von Krediten bei Drittweltstaaten zu ernsthaften Schwierigkeiten führte, gibt es auch Erfolgsgeschichten. Heute sind es v. a. einige asiatische Länder, die dank Krediten gewaltige Entwicklungsfortschritte erzielen. Historische Beispiele für erfolgreiche vorübergehende Verschuldung liefern die meisten Industrieländer. Zum vornherein kann man also nicht leicht entscheiden, ob eine Kreditaufnahme sinnvoll und verantwortbar ist. Nachträglich muß man zumindest jene Verschuldungsmodelle als entwicklungshemmend bezeichnen, die zu Verschuldungsproblemen- bzw. -krisen geführt haben. Dies war bei Mexiko - wir wir wissen - zweifellos der Fall.

Stefan Denzler, dipl. Geogr., Geograph. Institut, Universität Zürich-Irchel, Winterthurerstrasse 190, 8057 Zürich 


\section{Die Verschuldungskrise Mexikos: unvorhersehbar?}

Am 12. August 1982 telefoniert der mexikanische Finanzminister Silva Herzog nach Washington. Seine schockierende Nachricht: Mexiko gehen die Devisenreserven aus. Gerade 200 Mio. \$ liegen noch in der Kasse - bei einem Verbrauch von 100 Mio. täglich. Mit einem Milliardenkredit der USRegierung wird die erste Gefahr abgewendet, aber die Lage spitzt sich wiederum zu. Mexikos Präsident Portillo tritt mit der Verstaatlichung der Privatbanken die Flucht nach vorn an. Schließlich arrangiert der Präsident des Internationalen Währungsfonds (IWF) ein Kreditpaket von $8 \mathrm{Mia}$. \$ (Kraft 1984). Er verhindert so die Zahlungsunfähigkeit Mexikos und damit einen weltweiten Bankenkrach. $\mathrm{Daß}$ eine reale Gefahr eines solchen Crash bestand, wird heute von beteiligten Fachleuten, so vom ehemaligen Schweizer Nationalbankpräsidenten Fritz Leutwiler, offen zugestanden. Erstaunlich aus heutiger Sicht ist, wie überraschend - sowohl für die Bankenwelt wie für die Mexikaner - 1982 die Krise eintrat.

Eigentlich hätte es genügend Warnsignale gegeben, die aber in den Wind geschlagen wurden. Die vorgängigen Zahlungskrisen von Polen und Argentinien etwa wurden als Sonderfälle abgetan. Aber auch die Verschuldungsindikatoren Mexikos zeigten bedenkliche Trends an (Weltbank: World Debt Tables).

Mexiko hatte 1982 eine Explosion der öffentlichen Außenschuld hinter sich. Der Schuldenberg stieg nominal von 3 Mrd. \$ 1970 auf über 72 Mrd. 1985. Auch wenn wir Bevölkerungswachstum und Inflation ausklammern, resultiert für den selben Zeitraum noch eine beeindruckende Verfünffachung der realen Außenschuld pro Kopf.

Diese Größenordnung wird bestätigt, wenn wir den Indikator Schuldenrelation (Außenschuld dividiert durch BSP) heranziehen. Hier verzeichnet Mexiko einen sprunghaften Anstieg von 9,1\% 1970 auf 43,4\% 1985 und liegt damit deutlich über den $32 \%$ für die EL insgesamt. Die Außenschuld Mexikos nahm also im Vergleich zu seiner Wirtschaftskraft zu, und zwar überdurchschnittlich.

Die zunehmende Belastung der Exporteinnahmen durch den Schuldendienst, d. h. durch Zins- und Tilgungszahlungen, belegt die Schuldendienstrelation. Die krisenträchtige Entwicklung bis 1979 konnte zwar durch die Erdöleinnahmen gebremst werden. Trotzdem blieb Mexiko fast auf dem doppelten Wert wie die Entwicklungsländer insgesamt. Dies liegt u. a. daran, daß Mexikos Wirtschaft stark binnenmarktorientiert ist. Die Exporte machen nur $5-10 \%$ des BSP aus.

Woher bezog Mexiko seine Kredite? Die Aufteilung nach öffentlichen und privaten Geldgebern illustriert, wie Mexiko - anteilsmäßig - viel umfangrei- cher auf private Quellen zurückgreift als die Entwicklungsländer insgesamt. Der Privatanteil hat sich zwischen 1970 und 1985 von $62,5 \%$ auf $90 \%$ ausgedehnt. Damit verbunden ist natürlich eine Verschlechterung der Kreditbedingungen.

\section{Ursachen der Krise}

\section{a) Externe Faktoren}

1) Prebischs These der säkularen Verschlechterung der Terms of Trade (Commodity ToT: Exportpreisindex im Verhältnis zum Importpreisindex; vgl. wAGNER 1983) der Entwicklungsländer läßt sich für die siebziger Jahre nicht belegen. Prebisch ging davon aus, daß die Nachfrage nach Nahrungsmitteln und Rohstoffen bei Einkommenssteigerungen unterproportional bleibt - Einkommenselastizität unter 1-, somit die Preise dieser Produkte fallen und die Peripherie (Entwicklungsländer) einen Realeinkommenstransfer in den Zentren (Industrieländer) leistet (NOHLEN/NUSCHELER, 1982, 306f). Paradoxerweise verlaufen die ToT während der Phase des Schuldenaufbaus für die Entwicklungsländer, besonders für Erdölexporteure wie Mexiko, ausgesprochen günstig. Verständlich ist dagegen, daß der Erdölpreiseinbruch 1982, also eine Situation sinkender ToT, das wichtigste auslösende Moment der Schuldenkrise Mexikos war.

2) Als weiteres auslösendes Moment muß der Übergang von einer Phase negativer Realzinsen in eine Hochzinsphase erwähnt werden. Ende der siebziger und Anfang der achtziger Jahre betrieben die USA eine ausgeprägte Hochzinspolitik, um den seit 1977 drohenden Zerfall des Dollars aufzuhalten und das amerikanische Haushalts- und Handelsdefizit zu decken. Die USA traten damit zusehends in Konkurrenz mit den Entwicklungsländern um internationales Kapital.

Wenige Jahre zuvor hatten die EL noch Geld zu negativen Realzinsen aufnehmen können. Damals wurde quasi das Schuldenmachen durch den Kapitalmarkt subventioniert. Die Ursachen dieser ungewöhnlichen Negativzinsen sind einerseits im Transnationalisierungsproze $\beta$ der Banken zu suchen. Die sogenannten freien Bankzonen (Offshore- oder Euro-Finanzplätze) erlaubten eine viel raschere Zirkulation des internationalen Geldkapitals und ermöglichten günstigere Zinssätze für Gläubiger und Schuldner dank kleineren Margen der Banken (sCHUBERT, 1985). Die zweite Ursache bildete die Petro-Dollar-Schwemme. Vor allem der Konjunktureinbruch, welchen der Erdölpreisschock von 1973 in den Industrieländern hervorrief, veranlaßte die Unternehmer, Gewinne anderswo, beispielsweise in der Dritten Welt, anzulegen. Der erhöhte Konkurrenzdruck unter den Banken verleitete 
diese dazu, Gelder nicht mehr projektgebunden zu vergeben, sondern Ländern bzw. Regierungen Kredite zu deren freien Verwendung zu erteilen. Damit gaben die Banken eine wichtige Kontrollmöglichkeit preis.

Der rasche Übergang zu höheren Realzinsen verteuerte den mexikanischen Schuldendienst empfindlich, zumal die Mexikaner überdurchschnittlich viele Kredite mit variablem Zinssatz verzeichneten. Geht man von einer Brutto-Konzeption der Außenschuld aus, so war der Zinsanstieg weniger gravierend, weil auch die mexikanischen Auslandguthaben nun mehr Profit abwarfen.

3) Als dritter externer Faktor müssen die falschen Anreize des internationales Auffangnetzes für Schuldenkrisen genannt werden.

In der Therapie von Schuldenkrisen hat der IWF eine neue Aufgabe gefunden, nachdem die Einführung freier Wechselkurse ihn seiner ursprünglichen Rolle beraubt hatte. Ungeachtet der Stärken und Schwächen von IWF-Programmen muß an dieser Stelle auf ein grundlegenderes Problem hingewiesen werden. Der IWF fusktioniert im Prinzip wie eine Versicherung gegen Zahlungsunfähigkeit von Staaten. Wie alle Versicherungen ist er mit der unangenehmen Tatsache konfrontiert, da $\beta$ allein durch seine Existenz Anzahl und Umfang der «Schadenfälle» zunehmen (moral hazard). Banken vergeben zu leichtsinnig Kredite, und Schuldnerländer nehmen zu viele solche auf, wenn sie davon überzeugt sind, durch die Interventionen des IWF vor großem Schaden im Falle einer Zahlungskrise bewahrt zu bleiben.

4) Als Grenzbereich zwischen externer und interner Ursache gilt es (Natur-) Katastrophen zu erwähnen. Aus Entwicklungsländern vernimmt man besonders häufig Katastrophenmeldungen. Dies kann mit dem höheren Risiko, aber auch mit mangelnder Vorsorge und ineffizienter Bewältigung von Katastrophen erklärt werden. Mexiko liegt in einem tektonisch unruhigen Gebiet (an der Nahtstelle zwischen der amerikanischen, der pazifischen, der Cocosund der karibischen Platte).

Das tragische Erdbeben vom 19. September 1985 legte in drei Minuten Teile der Hauptstadt in Schutt und Trümmer. Tausende von Menschen fanden den Tod, über 50000 wurden obdachlos. Der Deviseneinnahmenverlust wurde später auf $400 \mathrm{Mio}$. $\$$ beziffert, davon 300 Mio. im Tourismus, der Totalschaden auf rund $4 \mathrm{Mrd}$. Die eigentliche Tragik bestand jedoch darin, daß ein Großteil der Toten sowie der Kosten und Folgekosten zu vermeiden gewesen wären: Sie beruhten auf fehlerhafter Bausubstanz, Verzögerungen des Wiederaufbaus und auf der bürokratisch-ineffizienten Verteilung mancher Hilfsgelder. Damit sind wir in der Grauzone zwischen internen und externen Ursachen angelangt.
Als unfallträchtiger Bereich gilt die staatliche Ölgesellschaft PEMEX. Bei verschiedenen Pannen, u. a. auf Bohrinseln, kamen zwischen 1982 und 1986 über 1000 Arbeiter von PEMEX oder ihren Vertragsgesellschaften ums Leben. Nebst den Produktionsausfällen wurden zudem weitreichende ökologische Schäden angerichtet (HAFKEMEYER, 1988).

Mit dem auch in Mexiko erkenntlichen Hang zum Gigantismus wurde auch die Wahrscheinlichkeit von Großkatastrophen drastisch erhöht. Die beiden Siedewasserreaktoren des Atomkraftwerkes Laguna Verde beunruhigen denn auch die Bevölkerung: nicht nur aus Kostengründen (3,5 Mia. $\$$ bisher statt der geplanten 500 Mio.), sondern aus Sicherheitsüberlegungen. Einerseits liegt das Kraftwerk nur $18 \mathrm{~km}$ von einem tätigen Vulkan entfernt, andererseits ist es bei den mangelhaften Servicearbeiten staatlicher Unternehmen fraglich, ob Mexiko die Nukleartechnologie sicher beherrschen wird.

Immerhin muß auch erwähnt werden, da $\beta$ Mexiko auch erfolgreich Katastrophen verhindert hat. Als größte Leistung ist dem System wohl anzurechnen, $\mathrm{da} \beta$ es seit der Revolution das Land von Bürgerkrieg verschont hat und zu einem Faktor makropolitischer Stabilität geworden ist (MOLS, 1981).

\section{b) Interne Faktoren}

5) Das risikoreiche Schuldenmanagement Mexikos, ein erster interner Faktor, wird durch das politische System begünstigt oder geradezu provoziert. Der Präsident wird in Mexiko für eine Dauer von sechs Jahren gewählt, ohne Recht auf Wiederwahl, und besitzt in seiner Amtszeit weitgehende Vollmachten (vgl. LEHR, 1981). Das System wurde darum auch schon als Demokratur tituliert. Nach dem Motto «Nach-mir-die-Sintflut» versuchten so die meisten Regierungen, zum Zwecke persönlicher Bereicherung die Verschuldungskapazität des Landes bis an die äußerste Grenze auszunützen.

Das riskante Schuldenmanagement hängt daneben mit dem in Mexiko praktizierten Entwicklungsmodell der "verschuldeten Industrialisierung" und dem Erdölboom zusammen. Nach ersten Funden Anfang des Jahrhunderts entwickelte sich Mexiko dank ausländischen Ölgesellschaften bis 1921 zum führenden Erölexporteur der Welt. Aufgrund eines Arbeitskonfliktes verstaatlichte Präsident Cardenas 1938 die Ölgesellschaften und gründete die PEMEX. Mexiko betrieb darauf eine Abkoppelungsstrategie bezüglich Öl, produzierte also nur noch für den Eigenbedarf. Erst in den siebziger Jahren setzte Mexiko wieder auf die Karte Export. Die immer größeren gesichteten Ölvorkommen ließen Mexiko kreditwürdig erscheinen und öffneten den $\mathrm{Zu}$ gang zu Krediten in fast beliebiger Höhe. Oft wurde sogar mit steigenden Ölpreisen kalkuliert (vgl. 
STANKIEwICZ, 1984). Diese Rechnung konnte ökonomisch nicht aufgehen, wie jedermann seit 1982 weiß.

6) Das permanente Haushaltdefizit ist der wohl deutlichste Ausdruck des mexikanischen "schwachen Staates», eines weiteren internen Faktors. Schwach in dem Sinne, als es dem Staat nicht gelingt, die benötigten Einnahmen über ein einigermaßen effizientes System von Steuern zu beschaffen.

Das Haushaltdefizit wird zu einem guten Teil durch die defizitären Staatsunternehmen verursacht. In Mexiko, einem typischen Fall einer «mixed economy», ist der Staat in fast allen Branchen auch unternehmerisch tätig. Das Schwergewicht der Staatsunternehmen liegt bei der Verarbeitung von Primärprodukten aus Land-, Forst- und Fischwirtschaft, bei Erdölförderung, Petrochemie und Elektrizität sowie im Erzbergbau und der Basismetallindustrie. Die Defizite entspringen erstens dem Preiszwang dieser Unternehmen: Sie müssen die nachgelagerte Industrie mit billigen Grundstoffen und Energie versorgen bzw. subventionieren. Die fixierten Preise begünstigen auch eine verzerrte Produktionsstruktur: zu energieintensiv, und damit auch zu kapitalintensiv (EHRKE, 1986). Zweitens entstehen die roten Zahlen der Staatsbetriebe wegen ihrer ineffizienten und korrupten Führung (siehe unten).

Die Deckung des Haushaltdefizits geschieht in $\mathrm{Me}$ xiko durch interne oder externe Verschuldung sowie über die Notenpresse. Durch die Notenbank kann so der Staat Geld ausgeben, welches er gar nicht erst zu erwirtschaften brauchte. Die logische Konsequenz ist eine andauernde Inflation, d. h. ein Anstieg des allgemeinen Preisniveaus.

Die Inflation ihrerseits leistet der zeitweiligen Überbewertung des Peso im Vergleich zum Dollar Vorschub, weil Mexiko häufig den Wechselkurs fix hält. Ein überbewerteter Peso wiederum behindert die Exporttätigkeit, erhöht demzufolge das Leistungsbilanzdefizit und damit die Verschuldung.

Ein weiteres Anzeichen des schwachen Staates liefert die in Mexiko weit verbreitete Korruption. Am Beispiel der Polizei skizziert Riding deren Mechanismus treffend (RIDING, 1986). Die Polizisten unterschlagen einen Teil der Bußengelder und genießen die soziale Sicherheit ihres Arbeitsplatzes, sind als Gegenleistung dafür zur Loyalität und zur Weitergabe der übrigen Einnahmen an ihre Vorgesetzten verpflichtet. Diese behalten wiederum einen Teil für sich und bezahlen dem nächsthöchsten Vorgesetzten Tribut usw.

Die Korruption ist somit ein ausgeklügeltes System, wie dem Staat zustehende Einnahmen in die Taschen Privater umgelenkt werden. Ein besonders anfälliger Bereich dafür ist die Erdölwirtschaft mit ihren Riesengewinnen. Diaz Serrano, der PEMEXDirektor der Ära Portillo, konnte schon 1980 den
Verbleib von 112 Mio. Faß Öl - das entsprach damals etwa 3,5 Mrd. \$ - nicht erklären. Es wird vermutet, daß Serrano insgesamt 10 bis $20 \mathrm{Mrd}$. $\$$ veruntreute (BUCHE, 1985).

Während man der Korruption eines kleinen Beamten noch einen positiven Verteilungseffekt zugutehalten kann, sind große Betrügereien der erwähnten Art sowohl einer entwicklungsfördernden $\mathrm{Di}$ stribution wie Allokation abträglich.

7) Einen dritten internen Faktor bildet das Vertrauensdefizit gegenüber den staatlich gesetzten Rahmenbedingungen. Die Korruption ist ein Aspekt des mangelnden Vertrauens der mexikanischen Bürger in ihren Staat. Aber auch die Banken und potentiellen ausländischen Investoren zeigen seit 1982 nur mehr wenig Vertrauen in die Fähigkeit des Staates, ökonomisch sinnvolle Rahmenbedingungen zu schaffen. Unsicherheit bringen die periodischen Richtungsänderungen der Wirtschaftspolitik. Sie erfolgen traditionell alle sechs Jahre nach der Vereidigung des neuen Präsidenten, der sich durch einschneidende Maßnahmen gegenüber seinem Vorgänger profilieren will.

Als Zeichen der Stärke und Konstanz ist es beliebt, den Wechselkurs stabil zu halten, wie gesagt mit der Folge periodischer Überbewertung des Peso. Eine Überbewertung kann als Subventionierung von privaten Devisenkäufen durch den Staat aufgefaßt werden. Die monatelange Weigerung, den Peso abzuwerten, kostete den mexikanischen Staat vor der Krise im August 1982 mehrere Mrd. \$ und war mit ein Grund für die leere Staatskasse (EHRKE, 1984). Die Angst vor Abwertungen war, neben Steuerflucht und Verdeckung illegaler Geschäfte - verschiedentlich Anstoß zur massiven Kapitalflucht aus Mexiko (LESSARD, 1987).

Die Morgan-Bank schätzt die Kapitalflucht von 1976 bis 1984 aus Mexiko auf 54 Mrd. \$ (BAUMER, 1987). Nach neueren Angaben rechnet Morgan mit Auslandguthaben von Mexikanern im Umfang von 84 Mrd. \$. Dies würde heißen, daß die Brutto-AuBenverschuldung der mexikanischen Nation nur rund $20 \mathrm{Mrd}$. \$ beträgt. Den staatlichen Verpflichtungen stehen weitgehend Guthaben Privater gegenüber. E. Iglesias, der ehemalige Direktor der UN-Wirtschaftskommission für Lateinamerika, kommentierte dieses auf dem ganzen Kontinent beobachtbare Phänomen in einem Vortrag am 5.2.1990 in Zürich mit der Bemerkung: «Lateinamerika ist verschuldet, nicht jedoch die Lateinamerikaner.»

\section{Das Zusammenspiel der Faktoren}

Die bislang isoliert dargestellten internen und externen Faktoren sind in Wirklichkeit komplex miteinander verbunden. Eine solche Beziehung besteht 
zwischen dem risikoreichen Schuldenmanagement und den Realzinsen: Steigende Realzinsen werden nur dann zum Problem, wenn ein großer Teil der Schulden (wie bei Mexiko) variable Zinsen aufweist. Eine weitere Beziehung läßt sich zwischen dem praktizierten Entwicklungsmodell und den Terms of Trade herstellen: Der sinkende Erdölpreis hatte in Mexiko darum so katastrophale Konsequenzen, weil sich Mexiko in eine gefährliche Exportabhängigkeit von einem einzelnen Gut (Monoexportstruktur) begeben hatte.

Trotz dieser Vorbehalte darf man die Frage stellen, inwieweit interne und inwieweit externe Ursachen für die Verschuldungskrise verantwortlich waren. Dabei liegen drei Schlüsse nahe:

1) Die internen Ursachen scheinen, so gut sich dies mit Zahlen überhaupt belegen läßt, eher bedeutender als die externen.

2) Die internen Faktoren sind eher langfristiger, struktureller Natur gewesen (sozioökonomisches System). Die externen Faktoren haben kurzfristig die zugrunde liegenden internen Ursachen verstärkt und damit den Anstoß zur Krise gegeben.

3) Will man nicht nur Symptome bekämpfen, sondern langfristig die Verschuldungsprobleme beseitigen, so muß man primär bei den internen Ursachen ansetzen.

\section{Krisenbewältigung: Probleme und Chancen}

Die Regierung de la Madrid (1982-88) verbrauchte einen Großteil ihrer Kräfte mit der Bewältigung der Schuldenkrise. Diese spielte sich in drei Phasen ab.

1) Vorerst ging es darum, den Zusammenbruch des internationalen Finanzsystems zu verhindern. Eine erste Soforthilfe im Umfang von 2 Mrd. \$ stellte die US-Regierung bereit. Danach schnürten die Zentralbanken der führenden westlichen Industrieländer (sog. Zehnergruppe oder G-10) unter Leitung der Bank für internationalen Zahlungsausgleich (BIZ) zusammen mit dem IWF ein Notkreditpaket, welches Mexiko erlaubte, seinen Verpflichtungen weiterhin nachzukommen. Diese Überwindung der akuten Zahlungsbilanzkrise durch Überbrückungskredite dauerte bis Mitte 1983.

2) In der zweiten Phase, ab 1983, wurden die Überbrückungskredite der BIZ durch länger dauernde Beistandskredite des IWF abgelöst. Die Gläubiger hatten etliche der internen Verschuldungsursachen erkannt, ohne jedoch darauf selbst Einfluß nehmen zu können. Der IWF dagegen knüpft seine Kredite bekanntlich an wirtschaftspolitische Auflagen (Konditionalität). Mit dem IWF ausgehandelte Strukturanpassungsprogramme zielen meist, so auch im Falle Mexikos, auf eine Reduktion des Bud- getdefizits und auf die Inflationsbekämpfung (vgl. Körner et al. 1984). Mexiko mußte demnach den Peso abwerten, den Mindestlohn senken, Subventionen streichen und die Importe kürzen. Diese Ziele ließen sich nur unter Inkaufnahme sozialer Härten erreichen. Die Reallöhne sind in Mexiko seit 1982 um über $50 \%$ gefallen.

Warum wurde diese Austeritätspolitik von Präsident de la Madrid trotz des absehbaren innenpolitischen Konfliktpotentials durchgeführt? Erstens entsprachen viele der angeordneten Maßnahmen offensichtlichen ökonomischen Notwendigkeiten. Tatsächlich war das Programm in einigen Bereichen äußerst erfolgreich. Die traditionell negative Handelsbilanz etwa konnte bereits 1983 in einen Überschuß von $14 \mathrm{Mrd}$. \$ verwandelt werden. Zweitens bildete ein IWF-Abkommen als eine Art Gütesiegel die Voraussetzung für nachfolgende Umschuldungsabkommen mit den Privatbanken. Ein IWF-Kredit konnte ein Mehrfaches an Folgekrediten anderer Institute auslösen. Die Rolle des IWF wurde darum auch als die eines Katalysators umschrieben. Dies ist insofern aber ziemlich unzutreffend, als die Geschäftsbanken bei den Umschuldungen kaum je Neugeld zur Verfügung stellten, sondern dies dem IWF überließen. Die Privatbanken wollten in erster Linie die Liquidität Mexikos zur Bedienung des Schuldendienstes aufrechterhalten. Die Amortisationsfristen wurden gestreckt, unter Einräumung mehrerer Freijahre, und allenfalls die Zinsen leicht ermäßigt. Diese Strategie machte so lange Sinn, als man das Schuldenproblem als Liquiditätskrise interpretierte.

3) 1985 begann eine dritte Phase, in der durch spezielle Programme und neue Finanzierungstechniken eine Lösung des Schuldenproblems angestrebt wurde. Der Baker-Plan wollte 15 stark verschuldeten Ländern, darunter Mexiko, aus der Krise und zu neuem Wachstum verhelfen, indem er die Zufuhr von mehreren Mrd. \$ an neuem Geld vorsah. Die Umsetzung dieses Programmes scheiterte weitgehend am Widerstand der Banken, welche sich weigerten, dem schlechten Geld noch gutes nachzuwerfen.

Verschiedene neue Finanzinstrumente wie Debt Equity Swaps, Exit Bonds oder Debt for Nature Swaps brachten Mexiko höchstens eine gewisse Erleichterung, nicht aber den erhofften Durchbruch in der Schuldenfrage. Immerhin konnte de la Madrid seinem Nachfolger Salinas eine mittelfristig stabilisierte Schuldensituation und sogar beachtliche Devisenreserven hinterlassen.

Wie sehen die Erfolgsaussichten der gegenwärtigen mexikanischen Regierung aus, das Schuldenproblem in absehbarer Zeit zu lösen? Ohne übertrieben optimistisch zu sein, kann man verschiedene Chancen für Mexiko erkennen: 


\section{1) Neue Entschuldungsmechanismen}

Wir scheinen uns momentan am Beginn einer neuen - vierten - Phase der Schuldenbewältigung zu befinden. Die Einsicht wächst, daß ohne mindestens partiellen Schuldenerlaß wenig zu erreichen ist. Die vom Baker-Nachfolger im Frühling 1989 präsentierte Brady-Initiative legt den Banken ein Menü mit drei Optionen vor:

* Teilerlaß (um 35\%) der Schulden und Garantie der vollen Zinsen auf die Restschuld

* Neugeld einschießen

* Absicherung der gesamten Schuld zu reduzierten Zinsen

Anscheinend tendieren die Banken am ehesten auf die dritte Variante und verzichten weitgehend auf die Optionen «Teilerlaß» und «Neugeld». Grund dafür dürfte u. a. das Trittbrettfahrerproblem sein: die beiden ersten Optionen würden v. a. nicht teilnehmenden Banken nützen, indem sie die Schuldenhöhe Mexikos abbauen und die Rückzahlungschancen der Mexiko-Kredite jener Banken verbessern. Um dem Problem der Trittbrettfahrer beizukommen, wurde u. a. vorgeschlagen, unter den Banken einen Zwang zum gemeinsamen Schuldenerlaß einzuführen (vgl. SACHS, 1989).

\section{2) Auftreten neuer Akteure}

In der Weltwirtschaft der achtziger Jahre ist eine interessante Gewichtsverlagerung unter den vorherrschenden Wirtschaftsgroßmächten zu beobachten. Auf der einen Seite entwickelten sich die USA vom wichtigsten Gläubigerland zu einem Kapitalimporteur und sind gegenwärtig weltweit das Land mit der größten Außenverschuldung (über $250 \mathrm{Mrd}$. \$; Ende 1985 bis Ende 1988: von 111 Mrd. \$ auf 533 Mrd. \$, «NZZ» vom 25.10.89, S. 33). Andererseits rückten Japan und die BRD auf die Ränge eins und zwei der Gläubigernationen vor (Netto-Auslandvermögen Japan Ende 1988: 292 Mrd. \$; BRD: 206 Mrd. \$).

Insbesondere der neue Akteur Japan könnte Mexiko zu einer Entspannung seiner Devisenknappheit verhelfen. Zunehmende japanische Direktinvestitionen in die Maquiladora (so nennt man die Fertigungsindustrie an der Grenze zu den USA) belegen deren Funktion als Sprungbrett in den Markt USA.

\section{3) Nachbar USA}

Als rettenden Strohhalm Mexikos gilt es schließlich die USA zu nennen, zu welchen die Mexikaner eine Art Hassliebe pflegen. Die USA werden als imperialistische Macht abgelehnt, spätestens seit sie Mitte des vorigen Jahrhunderts die Hälfte des mexikanischen Territoriums beschlagnahmten. Heute fürchtet man den Einfluß der amerikanischen Multis in Mexiko. Andererseits bewundern die Mexikaner den Wohlstand des großen Nachbarn. Millionen illegaler Einwanderer suchen jedes Jahr in den südlichen Staaten der USA ihr Glück.
Für die USA ist Mexiko ein geopolitisch vorrangiges Land. Neben dem Einwandererproblem seien nur die Stichworte «Drogenhandel», «Kommunismus in Zentralamerika» und «strategische Erdölreserven» genannt. Es verwundert darum auch nicht, daß die Amerikaner im Schuldendialog mit dem Baker- und dem Brady-Plan Initiativen ergriffen haben, die geradezu auf Mexiko zugeschnitten waren.

\section{4) Der neue Präsident Mexikos}

Von seiten der Unternehmer werden große Erwartungen in den 41jährigen Harvard-Absolventen gesetzt.

Politisch wird Mexiko wohl auch unter Salinas noch geraume Zeit keine Demokratie sein, wie die offensichtlich gefälschte Wahl Salinas' zum Präsidenten zeigt. Eine mexikanische Studie verweist auf den statistisch unwahrscheinlich hohen Anteil von Urnenergebnissen mit der Endziffer Null, auf die unrealistisch hohen Wählerzahlen einzelner Wahlkreise und auf Wahlkreise mit praktisch $100 \%$ PRIStimmen.

Wirtschaftlich geht Salinas aber weiter als all seine Vorgänger. Er privatisiert Unternehmen (Fluglinien; Teile der Grundstoffindustrie, Randbereiche der PEMEX), die vor kurzem noch als unantastbares Eigentum des Staates galten. Mit einigen spektakulären Festnahmen von Gewerkschaftsbossen (Ölarbeiter- sowie Lehrergewerkschaft) hat er nicht nur ein deutliches Zeichen gegen die Korruption gesetzt, sondern auch innenpolitisch seine Macht gefestigt. Außerdem hat Salinas die Import- und Exportrestriktionen fast vollständig abgebaut.

$\mathrm{Ob}$ diese Maßnahmen genügen, muß allerdings bezweifelt werden. Die mit dem Sozialpakt 1987 eingeführten Fixierungen von Preisen und Mindestlöhnen wurden noch nicht aufgehoben, was langfristig aber kaum zu umgehen sein wird. So verbirgt sich hinter der Jahresteuerung von $20 \%$ ein unberechenbares aufgestautes Inflationspotential. Das Haushaltdefizit wurde zwar reduziert, liegt aber immer noch über den Werten Brasiliens oder Argentiniens. Und schließlich hat der Staat in den letzten Jahren durch Emission immer neuer Schuldpapiere eine gewaltige Binnenschuld aufgebaut. Der Schuldendienst dafür belief sich 1988 auf 26 Mrd. \$ gegenüber 8 Mrd. für die Außenschuld. Damit erscheint der Handlungsspielraum für Salinas nicht mehr allzu groß.

Die Binnenschuld Mexikos wird in den nächsten Jahren eine zunehmende Bedeutung erlangen. Es ist zu hoffen, daß sie Präsident Salinas bei seinen Reformbestrebungen nicht behindert, sondern unterstützt. Dies könnte dann der Fall sein, wenn dank der Binnenschuld bedeutende Teile der mexikanischen Gesellschaft und Wirtschaft sich für einen gesunden, rückzahlungsfähigen Staat verantwortlich fühlen. Nur vereint mit diesen Kräften kann es Präsident Salinas gelingen, die skizzierten 
internen Krisenfaktoren auszuräumen und den Weg für eine anhaltende Entwicklung Mexikos zu ebnen.

\section{Literaturverzeichnis}

BAUMER, J.-M. (1987): Mexiko, Zürich.

BUCHE, I./METZGER, J./SCHELL, R. (Hrsg.) (1985): Mexiko: die versteinerte Revolution, Bornheim-Merten.

EHRKE, M. (1984): Spekulation und Auslandsverschuldung: Die Fälle Mexiko und Argentinien. In: Lateinamerika - Analysen und Berichte 8, Hamburg.

EHRKE, M. (1986): Der staatliche Sektor der mexikanischen Wirtschaft, Grüsch.

HAFKEMEYER, J. (1988): Mexiko: zwischen Maya und Moderne, Braunschweig.

KÖHN, D. (1984): Die Verschuldung lateinamerikanischer Länder, Hamburg.

KÖRNER, P., et al. (1984): Im Teufelskreis der Verschuldung: der Internationale Währungsfonds und die Dritte Welt, Hamburg.

KRAFT, J. (1984): The Mexican Rescue, New York.
LEHR, V. (1981): Der mexikanische Autoritarismus, München.

LESSARD, D.MILLIAMSON, J. (1987): Capital Flight and Third World Debt, Washington.

MOLS, M. (1981): Mexiko im 20. Jahrhundert, Zürich.

NOHLEN, D./NUSCHELER, F. (Hrsg.) (1982): Handbuch der Dritten Welt, Band 1, Hamburg.

NOHLEN, D. (Hrsg.) (1984): Lexikon Dritte Welt, Reinbek bei Hamburg.

RIDING, A. (1986): 18mal Mexiko, München.

SACHS, J. (1989): Making the Brady Plan Work. In: Foreign Affairs, Summer 1989.

SCHUBERT, A. (1985): Die internationale Verschuldung, Frankfurt am Main.

STANKIEWICZ, A. M. (1984): Mexiko - Erdöl und Entwicklungspolitik: Bestandesaufnahme, Kritik und Strategievorschlag, Diessenhofen.

VON GLEICH, A./GODAU, R./EHRKE, M. (Hrsg.) (1983): Mexiko: Der Weg in die Krise, Diessenhofen.

WAGNER, N./KAISER, M./BEIMDIEK, F. (1983): Ökonomie der Entwicklungsländer: eine Einführung, Stuttgart.

WELTBANK: Weltentwicklungsberichte 1985-89, Frankfurt. WELTBANK: World Debt Tables, Washington. 